\title{
Robust Fingerprint Matching Using Spiral Partitioning Scheme
}

\author{
Zhixin Shi and Venu Govindaraju \\ Center for Unified Biometrics and Sensors(CUBS) \\ State University of New York at Buffalo \\ Buffalo, NY 14228, USA \\ zshi@cedar . buffalo.edu \\ http://cubs.buffalo.edu
}

\begin{abstract}
Fingerprint matching for low quality or partial fingerprint images is very challenging. It is mainly because the features such as minutia points can not be extracted reliably. In the case of partial fingerprint images captured using solid state sensors, enough number of minutia points may not be included. In this paper, we introduce a novel fingerprint representation that combines information from each extracted minutia with detected ridges in its neighborhood. The proposed algorithm first enhances a fingerprint image and generates a binary image. Then instead of using thinning-based algorithms, the ridges are extracted using a chaincode scheme, which retains the original thickness of the ridges and precise local orientations. The minutia points are detected by tracing the ridge lines. Finally the enriched local structural features are built for each minutia by a spiral coding using the ridge line orientations around the minutia. The new features are translation and rotational invariant. Each feature vector represents a minutia and its neighboring ridge structures. Matching of two fingerprints is performed by calculating the Euclidean distances between pairs of corresponding feature vectors. Preliminary experiments show that the proposed algorithm is effective.
\end{abstract}

\section{Introduction}

A fingerprint is a graphical pattern of ridges and valleys on the surface of a human finger. Due to the uniqueness and permanence of fingerprints, they are among the most reliable human characteristics that can be used for personal identification 122. The uniqueness of a fingerprint is determined by the local ridge characteristics and their relationships [13. Ridge endings and ridge bifurcations, called minutiae, are generally considered to be the two most prominent characteristics. Most Automatic Fingerprint Identification Systems (AFIS) are based on minutiae matching. Each minutia is represented by attributes such as the type of the minutia point (ridge ending or bifurcation), its location and orientation. With detected minutia points, fingerprint matching is then reduced to a point matching problem. There are several minutia-based matching algorithms proposed in the literature. Besides approaches that apply direct point matching,

M. Tistarelli and M.S. Nixon (Eds.): ICB 2009, LNCS 5558, pp. 647-655 2009.

(C) Springer-Verlag Berlin Heidelberg 2009 
many minutia-based matching algorithms take into account the translational and rotational variations. A coupled approach is often used to match minutiae in clusters by constructing a translation and rotation invariant structure. A local structure composed of two or more minutiae close to each other serves to obtain minutiae correspondences 4 5 6 6/78]. These minutia-based matching approaches use only the minutia points and rely on the interdependencies between minutia details. The matching performance is evaluated by counting the matched minutiae.

Thus, the performance of a fingerprint verification/identification system using minutia-based approaches depends heavily on the performance of the corresponding minutia extraction algorithms. In practice, due to rotation and displacement of the finger placed on the sensor, there is often only a partial overlap between the template and query fingerprint images. This problem becomes more severe in the case of small solid-state sensors that are increasingly popular. Furthermore the fingerprint image quality is also affected by several factors such as the presence of scars, variations of the pressure between the finger and acquisition sensor, worn artifacts, and the environmental conditions during the acquisition process. Poor quality image affects the extraction of minutiae.

Another class of matching approaches are correlation-based using other features besides minutia points such as features from ridge orientations, phasebased features from Fourier transforms or statistic features from local intensity variations 910. These additional features provide more discriminative information. Matching these new features requires fingerprint registration[11]. But such alignments can only be obtained using other key features such as core points [9] or minutiae 11], which may be elusive. Typically, minutiae-based methods perform better than correlation-based ones.

In this paper, we propose a novel fingerprint representation that combines information from each extracted minutia with detected ridges in its neighborhood. The proposed algorithm first enhances a fingerprint image to generate a binary fingerprint image. Then instead of using thinning-based algorithms, the ridges are extracted using a chaincode scheme, which retains the ridges along with their original thickness and precise local orientations. The minutia points are detected by tracing the ridge lines. Finally, the enriched local structural features are built for each minutia by a circular coding using the ridge lines around the minutia. The new features are translation and rotational invariant. Each feature vector represents a minutia and its neighboring ridge structures. Matching of two fingerprints is by calculating the Euclidean distances between pairs of corresponding feature vectors.

The key aspects of our proposed representation are that the enriched minutiae features have stronger discriminative power and they can be used independently in matching fingerprints. The minutia and non-minutiae information extracted from the surrounding ridges are used together in building correspondences between fingerprints. In contrast to the minutia-based approaches 45678 , matching using the proposed representation requires fewer minutiae and therefore more practical with small images or partial images with small overlap areas. The 
proposed representation for one minutia is independent of that for any other minutia detected in the fingerprint hence it is more tolerant of erroneous outcomes of the minutia detection algorithm such as missing and spurious minutiae. The idea is about using rich local characterization for shape representation in order to reduce the complexity of feature correspondence and matching problems. The translation and rotation invariant properties also make the matching algorithm efficient.

Closely related to our approach is the work of Qi et al. [12] and Tico et al. 13 . Both use a sampling scheme to choose sample points in a circular neighborhood around a minutia and they record ridge orientations at the sample points. The feature vector is then constructed by the differences between the orientations at the samples points and the orientation of the center minutia. The sampling scheme employed by Qi et al. chooses sample points along three $120^{\circ}$ ray lines starting from a center minutia point. The sampling points in the work of Tico et al. are taken from a group of concentric circles. The distance between two points on the same circle is fixed. Minutiae extraction and ridge orientation field computation in both papers are thinning based and rely on an enhanced binary fingerprint image. Our minutia extraction is based on chaincode contours. The non-minutia features are extracted from the detected ridge lines which provide more reliable information than what can be obtained from the rough orientation field. For extracting ridge features, we apply a spiral tessellation scheme, which divides a neighborhood region around a minutia into sectors. Within each sector, ridge information is collected, which provides information that is more reliable than that collected from a number of random sampling points.

The circular tessellation is used in Jain's work 9 on reference points such as singular points to collect global features. Our spiral tessellation is constructed using an Archimedes' spiral centered at a minutia point. The spiral starts from the minutia's orientation direction. A number of ray lines starting from the center minutia point together with the spiral partition the neighborhood area of the minutia into sectors. The spiral tessellation is the key of our method, which makes the construction of translation and rotation invariant feature vectors possible.

The rest of the paper is organized as follows. Section 2 describes the minutia extraction algorithm used in our experiments. The definition of the proposed hybrid local structure and the matching algorithm are presented in section 3 . The experiment is presented in section 4. Finally, section 5 summarizes our work and provides pointers for future work along this direction.

\section{Chaincode-Based Image Enhancement and Minutia Extraction}

\subsection{Fingerprint Image Enhancement by Directional Filters}

An ideally sensed or scanned fingerprint image has clear and distinct ridges and valleys. For example, an ideal fingerprint image could be a rolled ink impression on a fingerprint card. The finger skin profile made of the ridges is evenly pressed 
on the flat paper card, which leaves the ink impression of ridges as continuous flow of foreground passes, and in between the ridges are the valleys as background with white color. But in practice, the fingerprint scanning devices are far from ideal. Even the NIST fingerprint images scanned from the inked fingerprint cards are not perfect. An image quality improvement algorithm is almost always needed. Our earlier work 14 employed a chaincode based approach in computing the ridge orientation field efficiently. The algorithm combines both the binary and the gray-scale image enhancement methods. A locally adaptive algorithm is used to obtain a binary fingerprint image of sufficient quality. The local direction field is estimated using a fast chaincode-base algorithm and a $15 \times 15$ mask, followed by applying an elliptically shaped filter [15] with its major axis aligned parallel to the local ridge direction. This increases the connectivity along the ridge direction.

\subsection{Minutiae Extraction Using Chaincode}

Most fingerprint minutia extraction methods are thinning-based where the skeletonisation process converts each ridge to one pixel wide. Minutia points are detected by locating the end points and bifurcation points on the thinned ridge skeleton based on the number of neighboring pixels.

Our chaincode based method is obtained by scanning the image from top to bottom and right to left. The transitions from white (background) to black (foreground) are detected. The contour is then traced counterclockwise and expressed as an array of contour elements. Each contour element represents a pixel on the contour. It contains fields for the $\mathrm{x}, \mathrm{y}$ coordinates of the pixel, the slope or direction of the contour into the pixel, and auxiliary information such as curvature.

In a binary fingerprint image, ridge lines are more than one pixel wide. Tracing a ridge line along its boundary in counterclockwise direction, a termination minutia (ridge ending) is detected when the trace makes a significant left turn. Similarly, a bifurcation minutia (a fork) is detected when the trace makes a significant right turn 14 .

The chaincode based algorithm extract minutiae and ridge lines much more reliably. The integrity of the ridge lines is preserved with respect to their locations and thickness. Figure 1 shows an example of minutia and ridge line result.

\section{Definition of the Proposed Hybrid Local Structure}

Regarding a shape as a discrete set of sample contour points, which propose a representation scheme that comprises of a coarse distribution of the shape with respect to each point. Since the contour sample points can be chosen in any number, one can always pick enough points in order to accurately represent the underlying shape. In contrast, the minutia points of a fingerprint capture only a very limited amount of information from the rich information content present in the fingerprint pattern. Consequently, a representation of the fingerprint pattern with respect to each minutia detail must employ non-minutia information like 


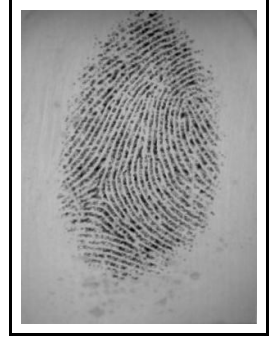

(a)

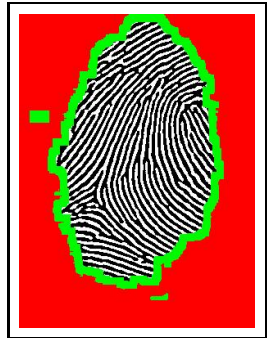

(b)

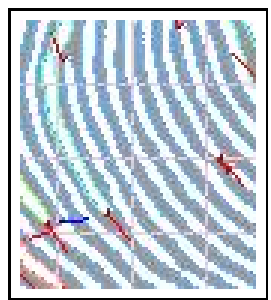

(c)

Fig. 1. Sample result showing fingerprint minutia and ridge line extractions

brightness, ridge orientation, transform features, etc. In this work, we investigate the use of orientation information for minutia representation in the context of the fingerprint pattern.

\subsection{Compute the Correlation Position for a Minutia}

We use an Archimedes' spiral centered at a minutia point, which is started from the minutia's orientation direction. Assume the angle between the orientation direction and the horizontal direction is $\theta_{0}$. Then the polar equation of the Archimedes' spiral is

$$
\rho=a\left(\theta-\theta_{0}\right)
$$

A number of ray lines starting from the center minutia point together with the spiral curve partition the neighborhood of the point. We draw $N$ ray lines that evenly divide the range of 0 to $2 \pi$, which give us $N$ sectors in each turn of the spiral (Figure 21). Since the first full turn covers the smallest area near the center, we start collecting features from the sectors starting from the second turn. The indices of the feature vector are arranged corresponding to the sectors along the spiral turning counter-clock-wise outward (Figure 3).

In the neighborhood of a center minutia covered by the spiral tessellation, if any other minutia point is included in a sector, the minutia contributes to the feature vector a strong correlation feature. The minutia information such as its location, orientation and type are added to the structure of the corresponding feature entry. Instead of searching every pixel in a sector for possible minutia points, we go with a more efficient approach to incorporating the surrounding minutiae in the feature vector.

For each such minutia point we compute its feature index that indicates which sector the minutia point belongs to. Assume that the current central minutia point is at $P_{c}=(x, y)$ and its orientation is represented by a vector $v_{c}=(X, Y)$ (Figure 3). For a minutia point at $P_{m}=\left(x_{m}, y_{m}\right)$, we compute a vector from $P_{c}$ to $P_{m}$ as $v_{m}=\left(X_{m}, Y_{m}\right)$, where $X_{m}=x_{m}-x$ and $Y_{m}=y_{m}-y$. To determine the angle $\theta_{m}$ between $v_{c}$ and $v_{m}$, we first compute

$$
\beta=\arccos \left(\frac{v_{c} \cdot v_{m}}{\left|v_{c}\right|\left|v_{m}\right|}\right)=\arccos \left(\frac{X X_{m}+Y Y_{m}}{\sqrt{X^{2}+Y^{2}} \sqrt{X_{m}^{2}+Y_{m}^{2}}}\right)
$$




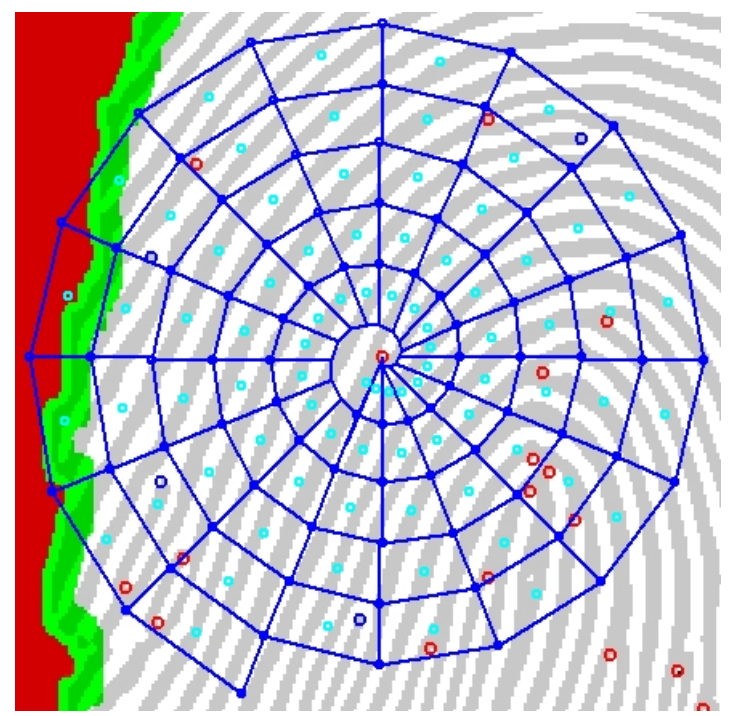

Fig. 2. Spiral tessellation around a minita point

and

$$
D=X Y_{m}-Y X_{m}
$$

Then the angle $\theta_{m} \in[0,2 \pi)$ is

$$
\theta_{m}=\left\{\begin{array}{cl}
\beta & \text { if } D \geq 0 \\
2 \pi-\beta & \text { if } D<0 .
\end{array}\right.
$$

If the distance between $P_{m}$ and $P_{c}$ is $d=\sqrt{\left(x_{m}-x\right)^{2}+\left(y_{m}-y\right)^{2}}$, and the successive turnings of the spiral have a constant separation distance of $2 a \pi$, then the index of the feature vector corresponding to the sector that $P_{m}$ belongs to is

$$
\text { index }=\left\lfloor\frac{d-a \theta_{m}}{2 a \pi}\right\rfloor N+\left\lceil\frac{\theta_{m}}{\Delta \theta}\right\rceil,
$$

where $\Delta \theta=\frac{2 \pi}{N}$. If we call the spiral the circular boundary of the sectors, and the sector including $P_{m}$ is $S_{m}$, then the first term is the index of the turn that makes the insider circular boundary of $S_{m}$. The second term is the angular index indicating which sector in the turn $P_{m}$ is in. We use the ceiling instead of floor in the second term because our regular indexing starts from 1 . The first feature entry indexed at 0 is from the entire inner area inside the first turn. Therefore around each minutia a feature vector is constructed which includes a list of indexed feature entries extracted from the above sectors. Since the features are calculated with respect to the center minutia, the features are rotation invariant.

\subsection{Add Local Orientation Features}

An orientation field is calculated for the fingerprint image before the constructions of each feature vector. When a feature vector is constructed around a center 


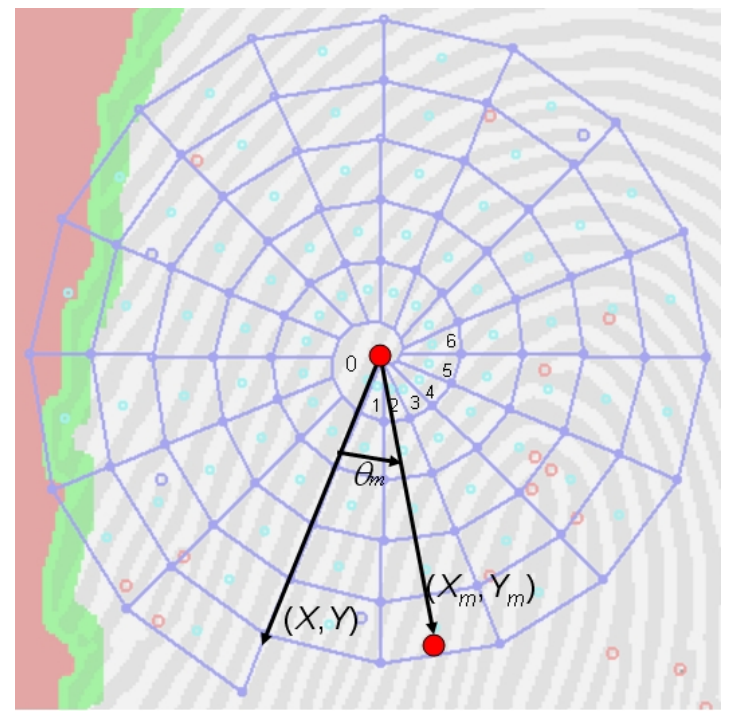

Fig. 3. Feature vector indexing arrangement and relative angle from a center minutia to another minutia point with respect to the direction of the center minutia

minutia, an average orientation direction in each sector of the spiral tessellation is calculated using the orientation field. The difference of the average orientation direction and the direction of the center minutia is the relative orientation of the sector, which is saved as one feature for the sector in the feature vector.

\subsection{Matching Two Structure Feature Vectors}

A Match of two fingerprints is by finding the best match of feature vectors of one fingerprint with those of the other fingerprint. Because the proposed feature vector construction is based on a spiral tessellation, a match between a pair feature vectors can be done by simply matching the features in the corresponding entries, which eliminates the need of an alignment between the two fingerprints. For each feature entry, the matching score is calculated from the matchings in the corresponding sector. The score is a weighted sum of the following values: (1) the number matched minutiae of same type over the total number of minutiae in the sector, (2) the difference between the orientations, and (3) overlapping sectors for common areas between two fingerprints.

\section{Experiment and Results}

Our algorithm has been implemented and tested primarily on the DB1 fingerprint databases of FVC2002 [16]. The DB1 database contains 110 different fingers and 8 impressions of each finger yielding a total of 880 fingerprints (388 pixels 374 pixels) at 500 dots per inch. The database has two different sets: A and 
B. Set A contains the fingerprint images from the first 100 fingers, while Set B has the images from the other 10 fingers. We use Set B of each database as our training set for matching parameters and then we perform the experiment on the fingerprints of Set A. We followed the protocols of FVC2002 [16] to evaluate the FAR (False Accept Rate) and FRR (False Reject Rate) of our system. For FRR, the total number of genuine tests (with no rejection) is $(87) / 2100=2800$. For FAR, the total number of false acceptance tests (with no rejection) is (100 x 99$) / 2=4950$.

The experiment is meant for showing the effectiveness of the new feature for fingerprint matching. We have achieved a $1.94 \%$ EER which is better than the result presented in [8] $(2.13 \%)$ and the NIST result presented in [17] $(3.35 \%)$.

\section{Conclusion}

We present a novel fingerprint matching algorithm based on hybrid local structural similarity. The local structural features are built around a detected minutia by combining the minutia information together with the local ridge orientation information. A feature structure is then constructed by sampling the minutiae and local ridge orientations using a spiral partition scheme. The new features are translation and rotational invariant. Each feature vector represents a minutia and its neighboring ridge structures. Matching of two fingerprints is done by calculating the distances between each pair of corresponding feature vectors from the two fingerprints. The final matching score is converted from the scores of the best matching pairs. We presented an experimental evaluation of the proposed approach and showed that the proposed feature structure is effective and it exceeds the performance of the earlier matching algorithms.

\section{References}

1. Lee, H.C., Gaensslen, R.E.: Advances in Fingerprint Technology. CRC Press, New York (1991)

2. O'Gorman, L.: Fingerprint verification. In: Jain, A.K., Bolle, R., Pankanti, S. (eds.) Biometrics-Personal Identification in Networked Society, pp. 43-64. Kluwer Academic, The Netherlands (1999)

3. Moenssens, A.: Fingerprint Technology. Chilton Book Company, London (1971)

4. Wahab, A., Chin, S., Tan, E.: Novel approach to automated fingerprint recognition. In: IEE Proceedings Vision, Image and Signal Processing, vol. 145(3), pp. 160-166 (1998)

5. Chen, Z., Kuo, C.H.: A topology-based matching algorithm for fingerprint authentication. In: 125th Annual IEEE Int. Carnahan Conference on Security Technology, pp. 84-87 (1991)

6. Ratha, N.K., Bolle, R.M., Pandit, V.D., Vaish, V.: Robust fingerprint authentication using local structural similarity. In: Fifth IEEE Workshop on Applications of Computer Vision, December 2000, pp. 29-34 (2000)

7. Chikkerur, S., Cartwright, A.N., Govindaraju, V.: K-plet and coupled BFS: A graph based fingerprint representation and matching algorithm. In: Zhang, D., Jain, A.K. (eds.) ICB 2006. LNCS, vol. 3832, pp. 309-315. Springer, Heidelberg (2006) 
8. Jea, T., Govindaraju, V.: A minutia-based partial fingerprint recognition system. Pattern Recognition 38(10), 1672-1684 (2005)

9. Jain, A.K., Prabhakar, S., Hong, L., Pankanti, S.: Filterbank-based fingerprint matching. IEEE Trans. Pattern Analysis and Image Processing 9(5), 846-859 (2000)

10. Ito, K., Nakajima, H., Kobayashi, K., Aoki, T., Higuchi, T.: A fingerprint matching algorithm using phase-only correlation. IEICE Trans. Fundamentals E87-A, 886894 (2004)

11. Ross, A., Jain, A., Reisman, J.: A hybrid fingerprint matcher. Pattern Recognition 36(7), 1661-1673 (2003)

12. Qi, J., Yang, S., Wang, Y.: Fingerprint matching combining the global orientation field with minutia. Pattern Recognition Letters 26(15), 2424-2430 (2005)

13. Tico, M., Kuosmanen, P.: Fingerprint matching using an orientation-based minutia descriptor. IEEE Trans. Pattern Analysis and Machine Intelligence 25(8), 10091014 (2003)

14. Shi, Z., Govindaraju, V.: A chaincode based scheme for fingerprint feature extraction. Pattern Recognition Letters 27(5), 462-468 (2006)

15. Greenberg, S., Aladjem, M., Kogan, D.: Fingerprint image enhancement using filtering techniques. Real-Time Imaging 8, 227-236 (2002)

16. Maltoni, D., Maio, D., Jain, A., Salil, P.: Handbook of Fingerprint Recognition. Springer, New York (2003)

17. Watson, C.I., Garris, M.D., Tabassi, E., Wilson, C.L., McCabe, R.M., Stanley, J.: User's guide to nist fingerprint image software 2 (nfis2). National Institute of Standards and Technology (2004) 